\title{
Solving the Multiple Traveling Salesman Problem Using Memetic Algorithm
}

\author{
Ahmad T. Al-Taani* ${ }^{*}$, Lubna M. Al-Afifi \\ Department of Computer Sciences, Yarmouk University, Irbid, Jordan \\ E-mail: ahmadta@yu.edu.jo
}

Received: 13 October 2021; Revised: 4 January 2022; Accepted: 10 January 2022

\begin{abstract}
The Multiple Traveling Salesman Problem (MTSP) is considered as an NP-complete problem due to the difficulty of finding the shortest tour between different cities with a set of constraints such as visiting each city once by one salesman. The solution tour represents the sum of all tours' costs performed by $n$ salesmen. In this research, we propose a novel approach to find different solutions for various instances of MTSP based on a memetic algorithm using Genetic Algorithm (GA) and Hill-Climbing (HC) algorithm. The experimental results proved that the memetic approach achieved promising results i.e., minimum total tour compared to other existing approaches.
\end{abstract}

Keywords: hill-climbing algorithm, local search, shortest tour, population-based approach, hybrid metaheuristics, genetic algorithm

\section{Introduction}

The Traveling Salesman Problem (TSP) is known as a classical combinatorial optimization problem that required a Hamiltonian path with a minimum cost which was found among several available solutions. On the other hand, TSP is considered as a complete problem, i.e., the deterministic approaches are not effective for solving a problem with polynomial time. Therefore, the researchers attempted to explore new approaches to solve this problem. Therefore, researchers proposed several non-deterministic approaches either approximation algorithms or heuristic approaches [1]. TSP has several variants which are discussed in the literature review such as TSP with the Drone (TSP-D) [2], TSP with Time Windows (TSPTW) [3], and Multiple Traveling Salesmen Problem (MTSP) [4].

The MTSP poses a vital challenge that requires additional computations due to a different number of salesmen. The solution of TSP is represented as a set of arranged cities according to specific sequences, while MTSP is required to organize the visiting cities according to specific structural constraints of the problem [5].

The key advantages that motivated the researchers to use local search approaches for solving computationally hard optimization problems are finding the reasonable solution of infinite or large search space in constant memory. Therefore, this paper presents a new approach to solve MTSP by exploiting two approaches that belong to the local search and population-based algorithm families i.e., GA and HC algorithm. Besides, MTSP seeks to decide a group of Hamilton paths without sub-tours, in which each city is visited by only one salesman [6]. The MTSP can be solved by minimizing the total distance traveled by all the salesmen and minimizing the maximum distance traveled by any

Copyright (C2022 Ahmad T. Al-Taani, et al.

DOI: https://doi.org/10.37256/aie.3120221206

This is an open-access article distributed under a CC BY license

(Creative Commons Attribution 4.0 International License)

https://creativecommons.org/licenses/by/4.0/ 
salesman to balance the workload among salesmen [7].

Many different approaches have been proposed to address MTSP including Ant Colony Optimization (ACO) and GA which belong to nature-inspired approaches. ACO approaches are used to produce solutions that use the 2-opt edge exchange approach to improve the solutions obtained. The basic concept of using ACO to solve the MTSP is the salesmen on their tours simulate or act like ants in their search for food, they go separately leaving a pheromone in the path they follow while searching for food [8].

The main contribution of this research is proposing a new approach for enhancing the results obtained by state-ofthe-art research for solving the MTSP. The new proposed approach is based on combining the GA with the HC to find good solutions for the MTSP that have a large search space. The new approach provides an additional step to the genetic approach to minimize the total traveled distance which means reducing the total cost for each tour and to accelerate the process of convergence. This provides an optimal solution for each used instance of MTSP regardless of the number of cities. Also, the proposed approach improves the generated initial population which is used as input for the HC algorithm.

In this study, we propose suggestions for solving the popular problem of MTSP using a memetic algorithm and $\mathrm{HC}$ algorithm. The memetic algorithm is a special kind of GA that finds out the shortest path between start and end stations for multiple salesmen within predefined constraints of the problem. At the same time, we consider the speed of convergence whereby salesmen distributed within a specific zone and determined the trip path for each one. The merit of the memetic algorithm is to exploit the advantages of both combination approaches; evolutionary and local search to achieve enhancement. The fundamental idea behind using $\mathrm{HC}$ as an extra operator is to generate a new solution better than that generated based on random ways in the traditional Genetic Approach (GA). In contrast, GA is used for overcoming the local search stuck problem of the $\mathrm{HC}$ algorithm. Hence, the proposed approach can be classified as a new variant of GA depending on the three operations. selection, crossover, and mutation in addition to $\mathrm{HC}$ as the fourth operation. Therefore, the memetic algorithm has enabled us to address large-scale MTSP inefficient performance.

Despite the multiplicity of existing approaches that are presented to address the MTSP, each of them has drawbacks as will be mentioned later in the literature review. Researchers proposed different approaches based on GA to enhance the solutions and to increase the efficiency of search due to the difficulty of the MTSP. So, we suggested the use of the memetic algorithm to solve the MTSP since the orders of magnitude are faster and more accurate than GA on some problems and the suggested optimization framework combines the advantages of both GA and the HC local search algorithm which is able it to navigate the search space of salesman problem in an efficient way. So, this study provides a significant contribution to solving the MTSP which is characterized by its ability to find good solutions for problems that have a large search space with limited resources. The proposed approach provides an additional step to the GA to find the shortest path, i.e., reducing the total cost for each tour and provides an optimal solution for each used instance regardless number of cities. Also, the proposed approach improves the generated input initial population using the HC algorithm by the GA. The GA feeds its output as an input to the HC algorithm to improve the solution of the MTSP problem.

The remainder of the paper is structured as follows. Previous works related to MTSP are reviewed and analyzed in Section 2. The proposed approach is discussed in Section 3. Experimental results are presented and discussed in Section 4. Finally, the conclusion drawn from this research and directions for future work are presented in Section 5.

\section{Previous works}

The importance of the MTSP as an NP-complete problem came from the vital role which is played in different realtime problems. Solving the MTSP requires transforming the problem into a single TSP or relaxation problem to reduce some of the constraints $[3,5]$.

The rationale used to select the literature sources in this study is based on the following criteria:

- Since the scope of the study is enhancing the results of currently proposed approaches for solving the MTSP, only literature-proposed solutions for the MTSP are selected.

- The selection criteria are based also on the venue and year of publications, only papers published in reputed journals and conferences published in the last five years are considered. 
- Another criterion is based on the authors' specialties and expertise in the scope of the study. All selected papers are for those experts and well-known scholars in MTSP.

The literature is categorized into two main themes based on the methodology used, meta-heuristic approaches and hybrid approaches.

\subsection{Meta-heuristic approaches}

In [9] and [10], two approaches are proposed to solve the colored TSP using hypergraphs and objective variable neighborhood search. Population insertion is performed first to generate candidates' solutions, and then GA is used to find the best solution. Experiments are conducted on 20 cases and showed promising results compared to previous approaches.

Alazzam et al. [11] solved the MTSP by a meta-heuristic technique called discrete Pigeon-Inspired Optimizer (PIO). The total cost of the shortest tour for active salesmen with load balancing is computed. The merit of this approach is the ability to find the solution based on pigeon operators, landmark, map, and compass operators. Map and compass operators depend on both the position and velocity of salesmen, but landmark used salesmen's landmark neighboring. The PIO was tested using five instances of the TSPLIB. The experimental results showed that the PIO outperformed all compared algorithms within the most used instances. The best results are obtained with 2 and 3 salesmen for all instances.

Gulcu and Ornek [12] proposed a swarm optimization approach to solve the MTSP. The solution utilizes two metaheuristic approaches; APSO and HAPSO whereby the former is based on a 2-opt approach while the latter utilizes the Greedy Randomized Adaptive Search Procedure (GRASP). The research is aimed to determine which method performs better; APSO or HAPSO by evaluating the path-relink features as well as swarm operators. The experimental setup utilizes five instances of the salesmen, and the results are compared with the genetic approach and ACO approach. Additionally, the experimental setup utilizes the TSP dataset obtained from the TSBLIB library. It can be concluded from the results that the HAPSO approach achieved good results compared to the APSO approach based on generating the optimal route to be used by the traveling salesmen.

In [13], an approach is suggested to enhance the population initialization process and the crossover operator using GA to solve the MTSP by using multi chromosomes with a randomly cut-off point to guarantee that no redundant into generated off-springs. The proposed crossover operator depends on the degree of expectation to be in the solution route. The approach followed four steps: (1) population generation (2) calculating fitness value for each tour (3) selecting the top 10 candidate solutions (tours) with their break-point, and for any two tours swap the first city and the last city; the remaining cities are left to generate new off-springs and so on until the position of a city in the left side is greater than the one in the right side, (4) finally, apply 2-opt optimal mutation operator. Two objective functions are used to evaluate the proposed approach: the first is to minimize the total traveling cost of $n$ salesmen while the second is to minimize the longest tour traveling cost by any salesman. It can be induced from the results that the proposed approach achieved promising results for the first objective function, but the second objective function did not achieve satisfying results.

Alaid and Mahmood [14] proposed a distributed hybrid model to solve the MTSP. The proposed approach is implemented as a web service and utilizes multiple machines to enhance the results. Essentially, the proposed framework works by transforming the MTSP into the normal TSP and then applying the ACO using varying degrees of pheromone exchange. The performance of the ACO is further enhanced through the utilization of a local search. The ACO approach is used to determine the next city to be chosen in the process of route construction. In this context, the ACO is applied using three different PE policies notably $0 \%, 25 \%$, and $50 \%$. The probabilistic approach using neighborhood information is used to determine the next city to be chosen. The experimental results are further compared with other approaches such as MACO, NMACO, and MGA. According to the authors, the use of neighborhoods produced better preformation in terms of cost reduction.

In [15], a Two-Phase Heuristic Approach (TPHA) is suggested to solve the MTSP by group cities depending on specific locations. The proposed approach also utilizes the GA approach and the roulette wheel to perform route planning. The elitist strategy is further used to improve the GA approach selection process. The TPHA method is tested and validated using a mobile guide system specifically designed for use by tourists in the Baidu area. The results are evaluated and compared with other popular approaches used to solve MTSP. The results showed that the TPHA achieved better results by minimizing the traveling distance. 
Junjie and Dingwei [16] proposed an ACO approach to solving the MTSP where the number of salesmen was randomly allocated within a given range. The speed of evaporation and the number of salesmen who used the connection in their tours are considered. The route selection is based on probability function and updating traverse direction. Achieved results were competitive i.e., the best route for a small-scale instance of MTSP is found. However, it was unable to find the best route for large-scale instances due to requiring a long time for generating a solution.

In [17], Latah suggested a combined approach using the K-means clustering approach and the revised version of the ACO approach to solve the MTSP. The clustering approach is used to group cities into clusters while the ACO is used to find the optimal route from one city to another. Further, the approach utilizes a double crossover operation to derive new and better routes. The experimental setup uses the TSPLIB dataset to compare basic ACO, modified ACO, and GA approaches performance. Also, the experimental results are compared among the three approaches with subsequent validation in clusters of 25,50, and 75 cities and clusters of 2, 3, and 4 salesmen, and indicated that the updated ACO produces better performance in the terms of minimum distance and the optimal route that traveling salesmen need to use.

\subsection{Hybrid approaches}

Deng et al. in [18] proposed the use of a modified GA based on cellular automata and simulated annealing algorithm to solve the TSP. First, the selection operation of the modified GA is applied, then the GA is combined with the simulated annealing algorithm to search for the best tour. The proposed approach is tested using 13 instances from the TSBLIB. Good results are achieved compared to the other three algorithms.

In [19], Dong et al. suggested an optimized hybrid GA with variable neighborhood search for solving the MTSP. Candidates' solutions are generated first, then the crossover and mutation operators are used with the variable neighborhood search for updating the solutions. Promising results are achieved compared to current approaches for solving the MTSP.

In [20], the use of the K-means with crossover ACO is proposed to solve the MTSP. The main idea was to divide cities into many clusters, and then apply crossover ACO for each cluster to find the best route for each salesman. To evaluate the approach, three instances (att48, pr76, rat99) of TSPLIB are used with a different number of salesmen $(2,3$, 4, and 8). Experimental results proved that better results are achieved compared with other approaches using the k-means or the ACO.

In [8], a hybrid approach based on a modified ant colony, 2-opt, and GA are suggested (AC-Opt-GA). Each approach is used for a specific task, e.g. MAC generated a set of possible solutions then both of 2-opt exchange approaches and GA approach enhanced the fitness of the final solution. The suggested approach is based on a set of control parameters to guide an initial direction of movement whereby a weight that represented pheromone amount. Then, MAC is used beside the heuristic probability function based on two factors; the weight of edge and traversing cost to generate a single feasible route. The authors have reported good experimental results compared to other proposed approaches.

Changdar [21] proposed an approach to solving the MTSP using a hybrid combination of the GA and ACO. The solution used the solid MTSP, applied in a bubbling environment. The ACO is used for the selection of routes while the GA is used to construct the routes used by the salesmen. Additionally, in the optimization of the proposed approach, a combination of two-point mutation and the cyclic crossover is used. The goal was to generate travel costs using multiple instances with varying numbers of cities in a fuzzy rough environment. The results showed that genetic ACO is more effective and delivers practical data sets in a bubbling environment.

GA and the One-by-one Revision of the Two Sides (GORTS) approach is proposed in [22] to solve the dynamic TSP. GORTS combines the advantage of the global search ability of GA with the property of fast convergence of the one-by-one revision of two sides to find an optimal solution for the dynamic TSP in a reasonable time. The TSBLIB dataset is used to test the effectiveness of the proposed approach. Experimental results showed the efficiency of the GORTS approach compared with some other heuristic algorithms for solving the dynamic TSP.

In [23], a new crossover operator for the GA used with a set of 16 local search operators is proposed to solve the TSP with a Drone (TSP-D). The proposed approach has achieved the two main objectives of the study; minimum operational cost and min-time.

Mohammadpour et al. in [24] proposed a hybrid approach called Hybrid Gravitational Emulation Local Search 
(HGELS) to find an optimal solution for the MTSP and to balance the cost of the solution path and the distance that salesmen passed through. Single-point crossover and two-point crossover are used to guarantee the diversity of chromosomes, also exchange mutation operators are applied to enhance the off-springs, and GELS is used for optimizing the population. To measure the performance of the proposed approach, 19 instances are used. According to the results, the proposed approach achieved higher best fitness values for 10 instances and the results on the remaining instances were fair while the GA approach achieved higher best fitness values on 4 instances.

\section{Research methodology}

The proposed memetic approach used the HC local search algorithm and the GA. HC algorithm is used as an extra operator to the basic GA operators. The GA first generates a random population, and then applies the selection, crossover, and mutation operators as improvement steps to produce a new population. A random selection process is applied to each newly generated population for entering the local search operator. In this study, we used the HC algorithm to improve the fitness of each selected individual. Figure 1 shows the overall design for the proposed approach. Each city in the graph has a location determined by a point represented by a pair of coordinates ( $\mathrm{x}$, y) used for calculating the distance between pairs of neighbor cities. These distances represent the cost of the arcs between them. For each salesman, the proposed memetic approach is applied to find the best tour and the minimum total traveled distance for each salesman.

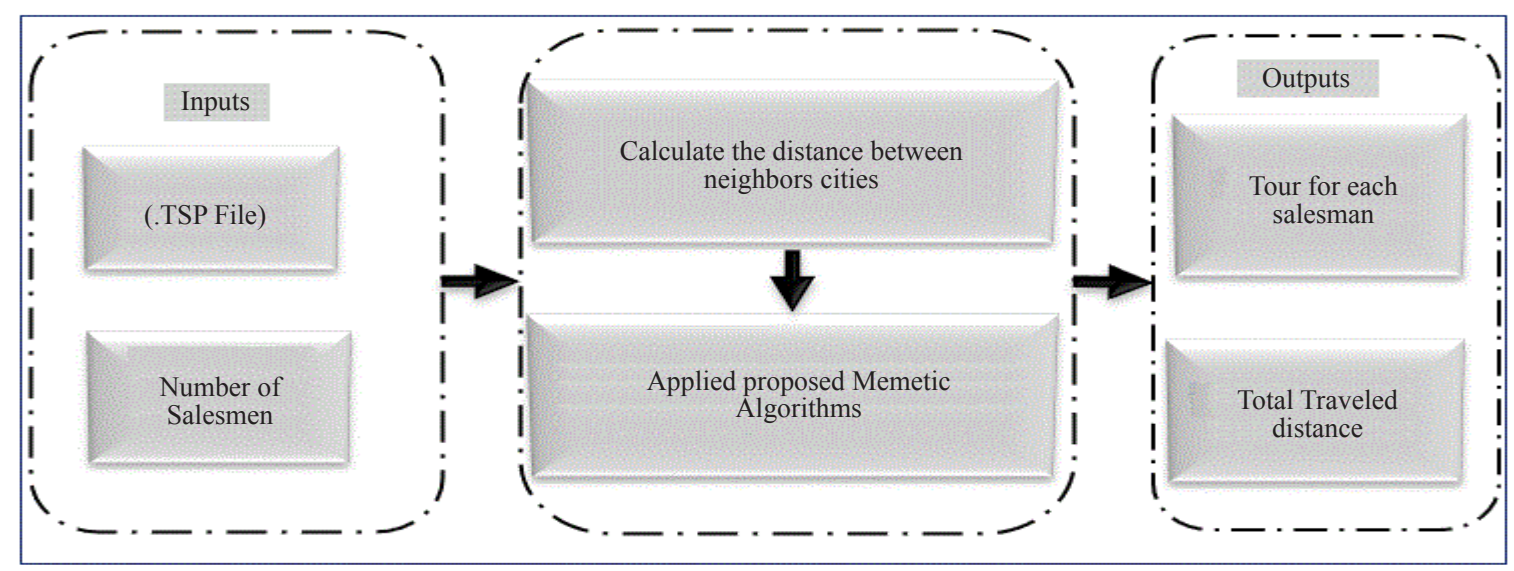

Figure 1. The architecture of the proposed approach

\subsection{Datasets}

The TSPLIB standard dataset is used for the experiments to show the advantage of the proposed memetic approach over existing approaches. The TSPLIB is a standard library consisting of several examples or instances for symmetric TSP which is represented as an undirected graph without loops. The nodes represent the set of cities and the edges show the whole possible paths between cities. The TSPLIB contains over 100 examples with sizes from 14 cities to about 86,000 cities. Each example represents the location of every city on the map as a pair of coordinates (x, y). We have selected some of the instances from the TSPLIB which are used in the previous studies for comparison purposes. Table 1 shows the characteristics of the dataset used in this research [23]. 
Table 1. Dataset proprieties

\begin{tabular}{lcc}
\hline No. & Instance & No. of cities \\
\hline 1 & Att48 & 48 \\
2 & Bayg29 & 29 \\
3 & berlin52 & 52 \\
4 & bier127 & 127 \\
5 & eil51 & 51 \\
6 & St70 & 70 \\
7 & Rat99 & 99 \\
8 & Eil101 & 101 \\
9 & pr76 & 76 \\
10 & Pr152 & 152 \\
11 & Pr144 & 144 \\
12 & Pr136 & 136 \\
13 & Pr124 & 124 \\
\hline
\end{tabular}

\subsection{The proposed memetic approach}

There are four main operators for the proposed memetic approach. The operators are used to guide the approach toward the solution for the problem. The first three operators of the memetic approach are the same as in the GA while the last one is used to accelerate the rate of convergence of the algorithm. The flowchart in Figure 2 shows the steps of the proposed memetic approach.

\subsubsection{The Genetic Algorithm (GA)}

The first task in the GA is to generate a suitable initial population, this process is mostly performed randomly. The initial population generation process is the first phase of any GA. For the TSP, the initial solutions i.e., initial tours are selected randomly [25]. There are three main operations in the GA, selection, crossover, and mutation. The selection operation is used for the chosen number of individuals from the population (current generation) for the next generation using crossover and mutation [26]. A Tournament selection is used for selecting the individuals for crossover, mutation, and local search operators. The selection of an approach mainly depends on the problem size [27]. The tournament selection method works by selecting many solutions or individuals randomly, selecting one of them based on their fitness value. According to Yadav and Sohal [28], tournament selection produces better results than rank-based selection since the selection process in the rank-based method requires sorting the population first and then assigning probabilities to them. Also, it is more efficient than proportionate roulette wheel selection in terms of convergence rate.

In the crossover operator, two solutions or individuals are selected by the tournament selection method, and then part of their solutions is swapped. There are two types of crossover operators such as one-point crossover or two-point crossover [26]. According to Soon et al. [29], a one-point crossover gives a better result than a two-point crossover for the TSP and is used in this study with rates between 0.6 and 0.9. Moreover, the obtained value of standard deviation for one-point crossover is less than the value obtained for two-point crossover.

The mutation operation is used to escape a locally optimal solution. The mutation is usually used by small rates ranging between 0.06 and 0.1 . The mutation operator is applied by swapping two index values randomly, where the 
quality of the GA solution depends on the efficiency of both the crossover and the mutation operators. The mutation operator keeps the diversity of the population by changing one or more genes randomly, where the crossover operator attempts to combine the advantages [29].

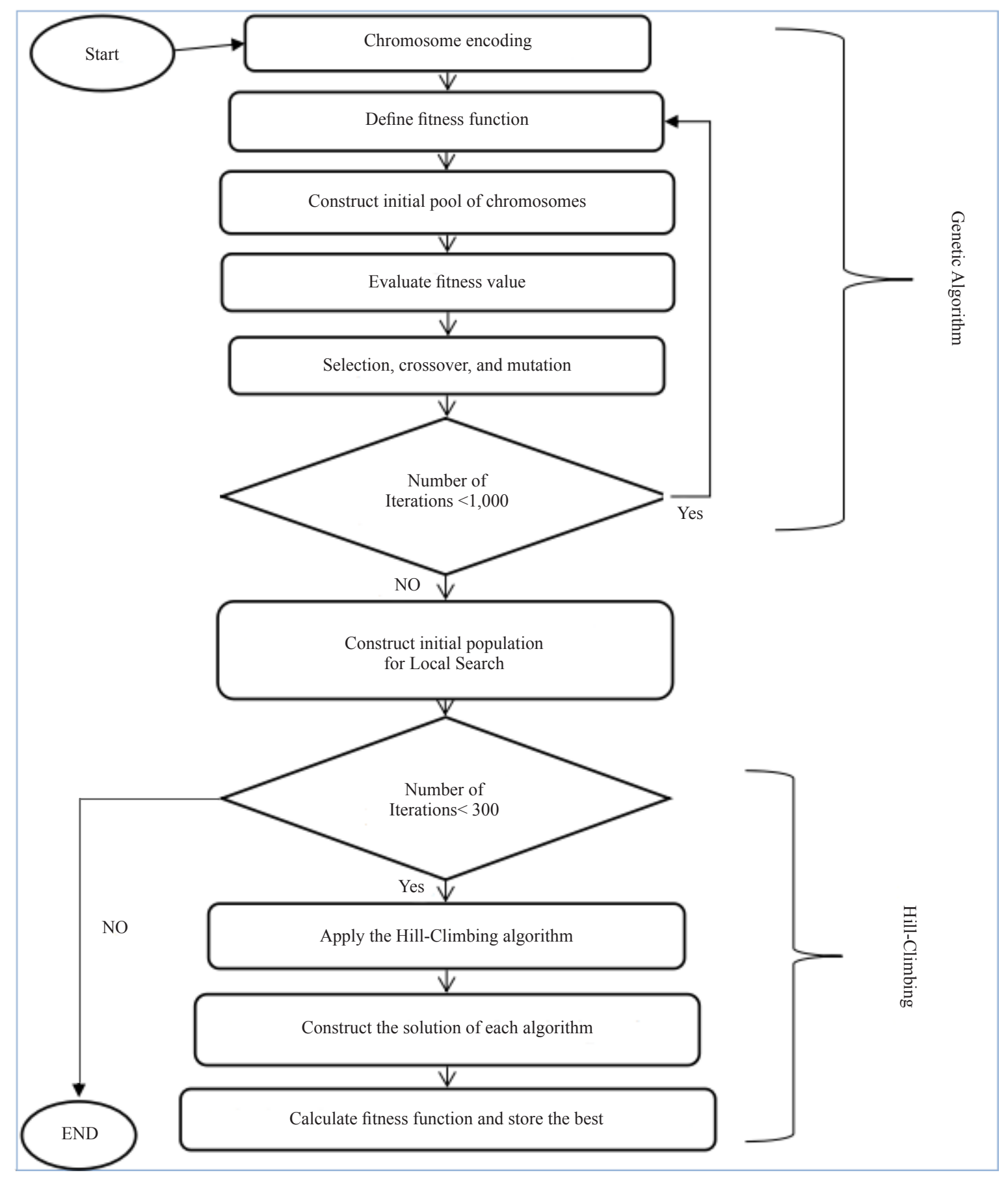

Figure 2. The steps of the proposed memetic approach

\subsubsection{The Hill Climbing (HC) algorithm}

The HC algorithm is used as an extra operator to the basic GA operators. It applied to some selected individuals 
from the population which was determined by the $\mathrm{HC}$ algorithm. The aim of using the $\mathrm{HC}$ algorithm is to accelerate the process of convergence. The HC is much faster than the basic GA. So, the combination of these two approaches generates a new approach faster than the basic approach. Moreover, the proposed approach can find the optimal solution since it uses the same operators of the GA alongside with $\mathrm{HC}$ algorithm.

\subsubsection{Fitness function}

Due to the nature of the MTSP and the variety of possible solutions, the quality of the solutions is evaluated using a reasonable fitness function based on the total distance of all salesmen trips. Moreover, the fitness function takes into consideration the load balancing between all salesmen trips. A fitness value is determined to determine chromosome's efficiency, when the fitness value is minimized, the optimal solution is sought [27]. In this paper, we aim to minimize the fitness function value, which depends on two main factors: the overall distance, and the load balance factor. The fitness function in Formulae 1-3 is used to evaluate the quality of the solutions in each population and to propose the candidate solutions.

$$
\text { Fitness Fun }=\left(\sum \text { Distance }(\mathrm{S} 1, \mathrm{~S} 2, \mathrm{~S} 3, \ldots, \mathrm{SN}) \times \text { Penality }(\mathrm{S} 1, \mathrm{~S} 2, \mathrm{~S} 3, \ldots, \mathrm{SN})+1\right)
$$

Where:

$$
\begin{gathered}
\text { distance }=\sqrt{(\mathrm{x} 1-\mathrm{x} 2)^{2}(\mathrm{y} 1-\mathrm{y} 2)^{2}} \\
\text { Penality }=\operatorname{std}(\text { number of } \operatorname{cities}(\mathrm{s} 1, \mathrm{~s} 2, \mathrm{~s} 3, \ldots, \mathrm{sn}))
\end{gathered}
$$

The optimal solution for the MTSP must achieve one objective function or more. Two objective functions are used in this study to evaluate the performance of the proposed memetic approach considering the constraints of the MTSP such as each city should be visited once and the initial station for all salesmen is the same. These objective functions are: minimizing the total travel distance of all salesmen's required tours (Formula 4) and minimizing the total traveled distance for each salesman's tour (Formula 5).

$$
\begin{gathered}
\operatorname{Minimize}\left(\sum_{\mathrm{k}=1}^{\mathrm{m}} \mathrm{C}(\mathrm{T})_{\mathrm{K}}\right) \\
\operatorname{Minimize}\left(\max \sum_{\mathrm{k}=1}^{\mathrm{m}} \mathrm{C}(\mathrm{T})_{\mathrm{K}}\right)
\end{gathered}
$$

Where $\mathrm{T}_{\mathrm{k}}$ denotes the tour traveled by the $\mathrm{k}^{\text {th }}$ salesman.

\section{Experimental results}

13 instances of the TSPLIB dataset are used for the evaluation of the proposed memetic approach. Each file represents a test case that includes coordinates of cities as $(x, y)$. These instances cover a different number of cities ranging from 29 to 152 according to scenarios that are mentioned in the dataset section. The proposed approach enables the user to assign the number of salesmen within 2 to 6 and the TSP instance to be used. 1,000 iterations for each instance are generated to investigate the effect of several iterations on the performance of the proposed memetic approach. The algorithm records three tours solutions with their total traveled cost for every single instance. 10 independent runs are conducted due to the randomness of the generation process of the initial population in the GA [30]. Then, the algorithm records an average case of obtained solutions for the pre-assigned number of salesmen within 2 to 6 . 
Table 2. Parameters setting

\begin{tabular}{cc}
\hline Parameter & Value \\
\hline No. of Iterations & 1,000 \\
Population size & 1,000 \\
Mutation rate & 0.05 \\
Memetic size & 300 \\
Memetic tries & Instance size \\
Tournament size & {$[2-6]$} \\
\hline
\end{tabular}

Table 3. Experimental results for 5 instances

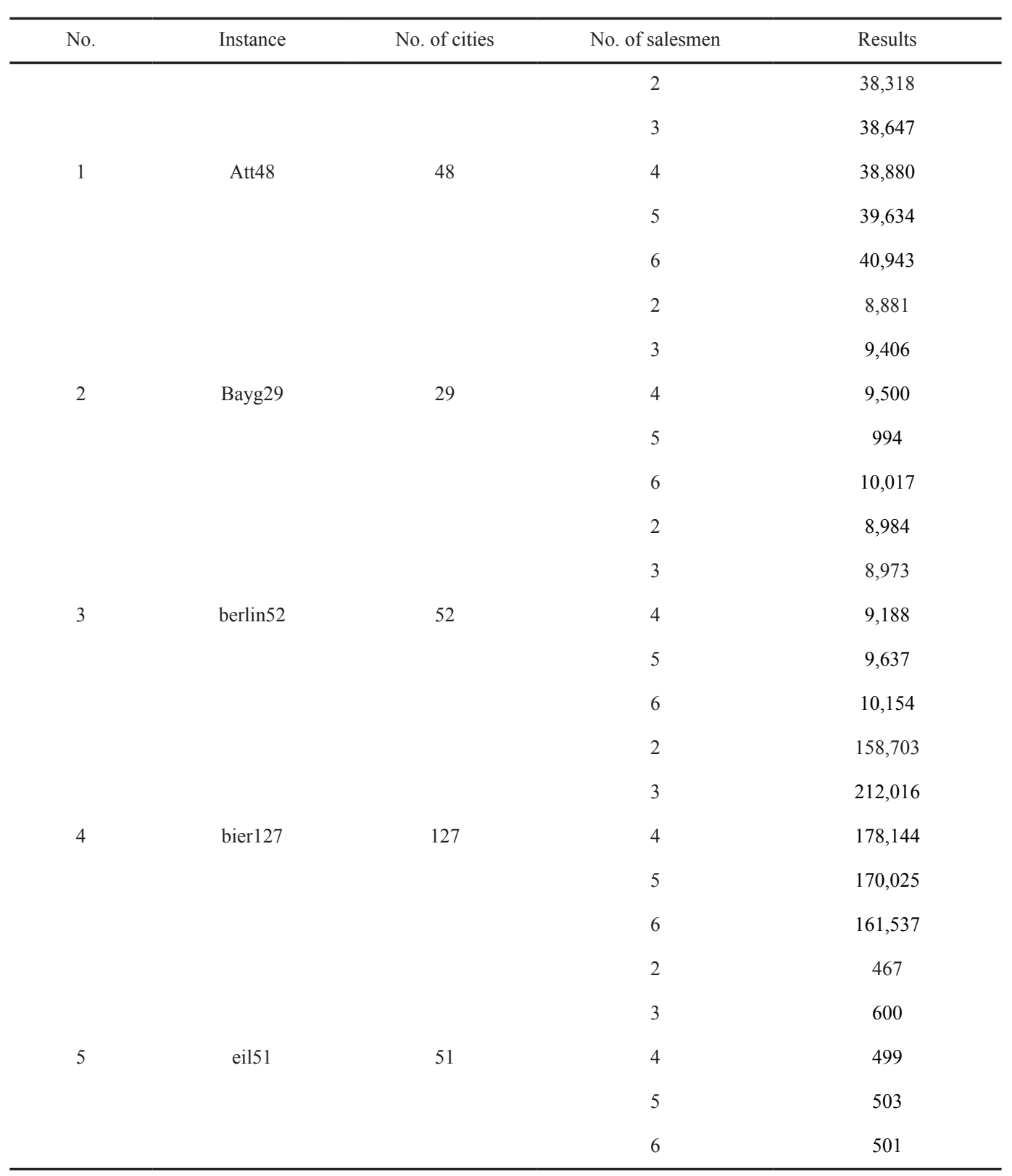


Table 4. Experimental results for 8 instances

\begin{tabular}{|c|c|c|c|c|}
\hline No. & Instance & No. of cities & No. of salesmen & Results \\
\hline & & & 2 & 971 \\
\hline & & & 3 & 1,041 \\
\hline \multirow[t]{5}{*}{1} & St70 & 70 & 4 & 1,021 \\
\hline & & & 5 & 1,072 \\
\hline & & & 6 & 1,062 \\
\hline & & & 2 & 5,365 \\
\hline & & & 3 & 1,839 \\
\hline \multirow[t]{5}{*}{2} & Rat99 & 99 & 4 & 1,927 \\
\hline & & & 5 & 1,970 \\
\hline & & & 6 & 2,025 \\
\hline & & & 2 & 901 \\
\hline & & & 3 & 941 \\
\hline \multirow[t]{5}{*}{3} & Eil101 & 101 & 4 & 920 \\
\hline & & & 5 & 980 \\
\hline & & & 6 & 943 \\
\hline & & & 2 & 150,615 \\
\hline & & & 3 & 154,047 \\
\hline \multirow[t]{5}{*}{4} & pr76 & 76 & 4 & 162,474 \\
\hline & & & 5 & 162,984 \\
\hline & & & 6 & 173,295 \\
\hline & & & 2 & 189,281 \\
\hline & & & 3 & 397,530 \\
\hline \multirow[t]{5}{*}{5} & $\operatorname{Pr} 152$ & 152 & 4 & 225,065 \\
\hline & & & 5 & 224,794 \\
\hline & & & 6 & 224,210 \\
\hline & & & 2 & 147,347 \\
\hline & & & 3 & 175,469 \\
\hline \multirow[t]{5}{*}{6} & $\operatorname{Pr} 144$ & 144 & 4 & 180,284 \\
\hline & & & 5 & 164,243 \\
\hline & & & 6 & 180,696 \\
\hline & & & 2 & 170,124 \\
\hline & & & 3 & 179,295 \\
\hline \multirow[t]{5}{*}{7} & $\operatorname{Pr} 136$ & 136 & 4 & 189,190 \\
\hline & & & 5 & 185,067 \\
\hline & & & 6 & 187,611 \\
\hline & & & 2 & 170,954 \\
\hline & & & 3 & 168,439 \\
\hline \multirow[t]{3}{*}{8} & $\operatorname{Pr} 124$ & 124 & 4 & 172,917 \\
\hline & & & 5 & 175,733 \\
\hline & & & 6 & 174,731 \\
\hline
\end{tabular}


Table 2 shows the general parameters setting used by the proposed memetic approach for the experiments. The mutation rate is determined experimentally. Other parameters were used as suggested by [30].

Achieved results for each instance are given in Table 3 and Table 4. The results show that the memetic approach using $\mathrm{HC}$ achieved competitive results. It can be noticed that for the first fourth instance in Table 3, the best cost of solution tour is achieved when the number of salesmen is 2 or 3 . For example, the best solution tour for the instance "berlin52" achieved with 2 salesmen even though the number of cities is quite large.

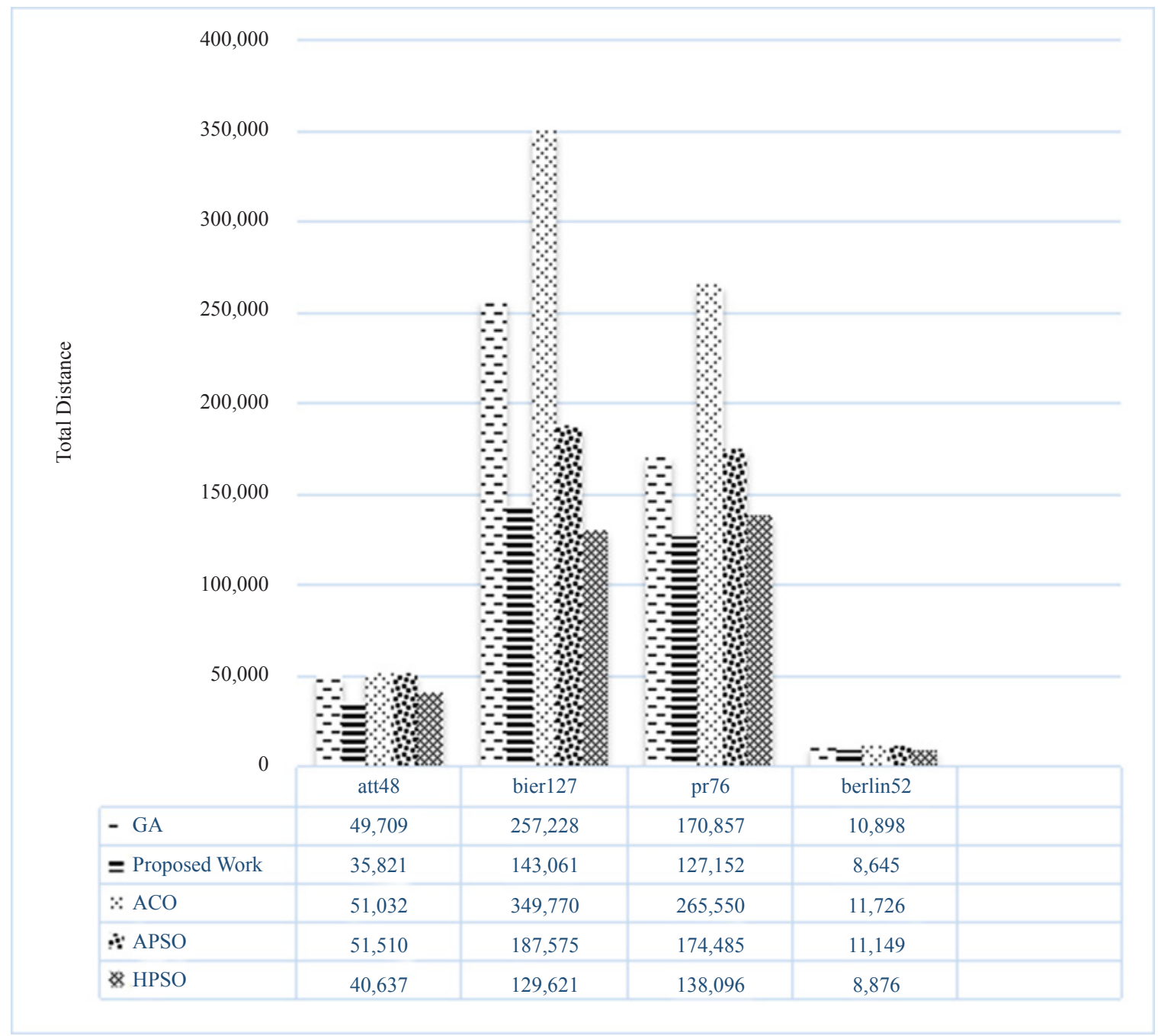

Figure 3. Comparison with other approaches

To show the advantage of the proposed approach over existing approaches, we compared achieved results with three recent approaches applied on five instances chosen from the TSPLIB dataset for three salesmen. These approaches used Adaptive Particle Swarm Optimization (APSO), Hybrid Particle Swarm Optimization (HPSO) [12], and Ant Colony Optimization (ACO) [17]. As can be noticed from Table 5 and Figure 3, the proposed approach achieved competitive results and small error rates (err) compared to those approaches. The error rate indicates the accuracy of the obtained results. The small error rate means the obtained solution is closed to the optimal solution [13]. The average error rate for the proposed approach is the smallest which is equal to 0.17488 compared to other approaches. The error rate (err) is calculated using Formula 6 below. 


$$
e r r=\frac{\omega(\text { best })-\omega(O H C)}{\omega(O H C)}
$$

Where best represents the best-obtained solution, and $O H C$ is the best-known solution.

The results indicate that the memetic approach achieved the best results compared with the recent approaches which satisfy the main objective of this study, finding the shortest trip for the MTSP.

Table 5. Comparison with other approaches

\begin{tabular}{|c|c|c|c|c|c|c|c|c|c|}
\hline \multirow[b]{2}{*}{ Instance } & \multirow[b]{2}{*}{$\begin{array}{c}\text { Optimal } \\
\text { solution } \\
(\mathrm{OHC})\end{array}$} & \multicolumn{2}{|c|}{ Proposed approach } & \multicolumn{2}{|c|}{$\mathrm{ACO}$} & \multicolumn{2}{|c|}{ APSO } & \multicolumn{2}{|c|}{ HPSO } \\
\hline & & $\begin{array}{l}\text { Obtained } \\
\text { solution } \\
\text { (Best) }\end{array}$ & Error Rate & $\begin{array}{l}\text { Obtained } \\
\text { solution } \\
\text { (Best) }\end{array}$ & Error Rate & $\begin{array}{l}\text { Obtained } \\
\text { solution } \\
\text { (Best) }\end{array}$ & Error Rate & $\begin{array}{l}\text { Obtained } \\
\text { solution } \\
\text { (Best) }\end{array}$ & Error Rate \\
\hline Rat99 & 1,211 & 1,626 & 0.3428 & 3,328 & 1.7481 & 2,018 & 0.6664 & 1,680 & 0.3873 \\
\hline Att 48 & 10,628 & 35,821 & 0.000317 & 51,032 & 3.8017 & 51,510 & 3.8466 & 40,637 & 2.8236 \\
\hline Bier127 & 118,282 & 143,061 & 0.2095 & 349,770 & 1.9571 & 187,575 & 0.5858 & 129,621 & 0.0986 \\
\hline $\operatorname{Pr} 76$ & 108,159 & 127,152 & 0.1756 & 265,550 & 1.4552 & 174,485 & 0.6132 & 138,096 & 0.2767 \\
\hline
\end{tabular}

It can be deduced from the experimental results that the memetic approach has achieved promising results compared with the state-of-the-art approaches for solving the MTSP. For instance, it can be concluded from Table 5 and Figure 3 that the proposed approach has outperformed the results of the four recent approaches for the four instances except for the HPSO approach for the Bier127 instance.

\section{Conclusion}

The MTSP is an extended version of the TSP which is classified as an NP-complete problem. The main idea is to find the tours for $m$ salesmen to visit all cities with least cost besides visiting each city exactly once.

The proposed memetic approach considers the workload balance factor according to two objective functions; minimizing the sum of the cost required tours and minimizing the traveled distance for each salesman's tour (shortest path) for solving the MTSP.

To evaluate the proposed approach, we used a set of instances of TSPLIB. The experimental results show that using the HC local search algorithm with the GA improves the performance and has a clear impact on the general performance of the proposed approach. Also, we noticed that the number of active salesmen is not correlated to the number of cities in an instance. In simple words, a large number of cities does not always require a large number of salesmen to obtain less total distance. The distance between neighboring cities is an essential factor.

The experimental results approved that the effectiveness of the proposed approach is not related to the number of active salesmen but the distribution of the cities in the solved instance. The proposed memetic approach could be used to solve similar problems such as the postman problem either single or multiple objectives.

For future work, we are planning to investigate the use of different local search algorithms such as beam search and simulated annealing and compare the results with the proposed approach. Also, we are planning to investigate the impact of using different crossover operators on overall performance. Also, we going to use another population-based optimization approaches such as ACO instead of GA. 


\section{Conflict of interest}

The authors declare that they have no conflict of interest.

\section{References}

[1] Rafaqat MK, Li JP, Kumar HK. Fluid flow algorithm to solve travel salesman problem. International Journal of Computing and Related Technologies. 2017; 1(1): 79-85.

[2] Kim S, Moon I. Traveling salesman problem with a drone station. IEEE Transactions on Systems, Man, and Cybernetics: Systems. 2019; 49(1): 42-52.

[3] Boland N, Hewitt M, Vu DM, Savelsbergh M. Solving the traveling salesman problem with time windows through dynamically generated time-expanded networks. In: Salvagnin D., Lombardi M. (eds). Integration of AI and OR Techniques in Constraint Programming. CPAIOR 2017. Lecture Notes in Computer Science. Springer, Cham; 2017. p. 254-262. Available from: doi: 10.1007/978-3-319-59776-8_21.

[4] Bektas T. The multiple traveling salesman problem: an overview of formulations and solution procedures. Omega. 2006; 34(3): 209-219. Available from: doi: 10.1016/j.omega.2004.10.004.

[5] Kaempfer Y, Wolf L. Learning the multiple traveling salesmen problem with permutation invariant pooling networks. ArXiv. 2018. Available from: doi: arXiv:1803.09621v2.

[6] Deng Y, Liu Y, Zhou DY. An improved genetic algorithm with initial population strategy for symmetric TSP. Mathematical Problems in Engineering. 2015; 2015: 1-6. Available from: doi: 10.1155/2015/212794.

[7] Venkatesh P, Singh A. Two metaheuristic approaches for the multiple traveling salesperson problem. Applied Soft Computing. 2015; 26: 74-89. Available from: doi: 10.1016/j.asoc.2014.09.029.

[8] Harrath Y, Salman A, Alqaddoumi A, Hasan H, Radhi R. A novel hybrid approach for solving the multiple traveling salesmen problem. Arab Journal of Basic and Applied Sciences. 2019; 1(26): 103-112.

[9] Xu XP, Li J, Zhou MC. Bi-Objective colored traveling salesman problems. IEEE Transactions on Intelligent Transportation Systems. 2021. Available from: doi: 10.1109/TITS.2021.3086625.

[10] Xu XP, Li J, Zhou MC. Delaunay-triangulation-based variable neighborhood search to solve large-scale general colored traveling salesman problems. IEEE Transactions on Intelligent Transportation Systems. 2021; 22(3): 15831593. Available from: doi: 10.1109/TITS.2020.2972389.

[11] Alazzam H, Alsmady A, Mardini W. Solving multiple traveling salesmen problem using discrete pigeon inspired optimizer. In: 2020 11th International Conference on Information and Communication Systems (ICICS). 2020. p. 209-213. Available from: doi: 10.1109/ICICS49469.2020.239528.

[12] Gulcu SD, Ornek HK. Solution of multiple travelling salesman problem using particle swarm optimization based algorithms. International Journal of Intelligent Systems and Applications in Engineering. 2019; 7(2): 72-82.

[13] Singh DR, Singh MK, Singh T, Prasad R. Genetic algorithm for solving multiple traveling salesmen problem using a new crossover and population generation. Computación y Sistemas. 2018; 22(2): 491-503. Available from: doi: $10.13053 /$ cys-22-2-2956.

[14] Alaidi AH, Mahmood A. Distributed hybrid method to solve multiple traveling salesman problems. International Conference on Advance of Sustainable Engineering, and its Application (ICASEA). 2018. p. 74-78. Available from: doi: 10.1109/ICASEA.2018.8370959.

[15] Xu X, Yuan H, Liptrot M, Trovati M. Two phase heuristic algorithm for the multiple-travelling salesman. Soft Computing. 2018; 22(19): 6567-6581. Available from: doi: 10.1007/s00500-017-2705-5.

[16] Pan JJ, Wang DW. An ant colony optimization algorithm for multiple travelling salesman problem. First International Conference on Innovative Computing, Information and Control. 2006. p. 210-213. Available from: doi: 10.1109/ICICIC.2006.40.

[17] Latah M. Solving multiple TSP problem by K-Means and crossover based modified ACO algorithm. International Journal of Engineering Research \& Technology (IJERT). 2016; 5(2): 430-434.

[18] Deng Y, Xiong J, Wang Q. A hybrid cellular genetic algorithm for the traveling salesman problem. Mathematical Problems in Engineering. 2021; 1-16. Available from: doi: 10.1155/2021/6697598.

[19] Dong XS, Zhang H, Xu M, Shen FF. Hybrid genetic algorithm with variable neighborhood search for multiscale multiple bottleneck traveling salesmen problem. Future Generation Computer Systems. 2021; 114: 229-242. Available from: doi: 10.1016/j.future.2020.07.008.

[20] Kusumahardhini N, Hertono GF, Handari BD. Implementation of K-Means and crossover ant colony 
optimization algorithm on multiple traveling salesman problem. Journal of Physics: Conference Series, Basic and Applied Sciences Interdisciplinary Conference 2017. 2020; 1442(1). Available from: doi: 10.1088/1742$6596 / 1442 / 1 / 012035$.

[21] Changdar C, Pal RK, Mahapatra GS. A genetic ant colony optimization-based algorithm for solid multiple travelling salesmen problem in fuzzy rough environment. Soft Computing. 2017; 21: 4661-4675. Available from: doi: 10.1007/s00500-016-2075-4.

[22] Xu XP, Yuan H, Mathew P, Ray J, Bagdasar O, Trovati M. GORTS: genetic algorithm based on one-by-one revision of two sides for dynamic travelling salesman problems. Soft Computing. 2020; 24: 7197-7210. Available from: doi: 10.1007/s00500-019-04335-2.

[23] Ha QM, Deville Y, Pham QD, Ha MH. A hybrid genetic algorithm for the traveling salesman problem with drone. Journal of Heuristics. 2020; 26(2): 219-247. Available from: doi: 10.1007/s10732-019-09431-y.

[24] Mohammadpour T, Yadollahi M, Bidgoli A, Esmaeelzadeh RH. HGELS: A new hybrid algorithm based on gravitational force for solving multiple traveling salesman problems. Journal of Advances in Computer Research. 2016; 7(3): 131-142.

[25] Hassanat AB, Prasath VB, Abbadi MA, Abu-Qdari SA. Faris H. An improved genetic algorithm with a new initialization mechanism based on regression techniques. Information. 2018; 9(7): 167. Available from: doi: 10.3390/info9070167.

[26] Kramer O. Genetic Algorithm Essentials. Studies in Computational Intelligence. Springer; 2017.

[27] Luo H, Xu W, Tan Y. A discrete fireworks algorithm for solving large-scale travel salesman problem. IEEE Congress on Evolutionary Computation (CEC). 2018. p. 1-8. Available from: doi: 10.1109/CEC.2018.8477992.

[28] Yadav LS, Sohal A. Comparative study of different selection techniques in genetic algorithm. International Journal of Engineering, Science and Mathematics. 2017; 6(3): 174-180.

[29] Soon GK, Guan TT, On CK, Alfred R, Anthony P. A comparison on the performance of crossover techniques in video game. 2013 IEEE International Conference on Control System, Computing and Engineering. 2013. p. 493498. Available from: doi: 10.1109/ICCSCE.2013.6720015.

[30] Yusoff M, Roslan N. Evaluation of genetic algorithm and hybrid genetic algorithm-hill climbing with elitist for lecturer university timetabling problem. In: Tan Y., Shi Y., Niu B. (eds.) Advances in Swarm Intelligence. ICSI 2019. Lecture Notes in Computer Science. Springer, Cham; 2019. p. 363-373. Available from: doi: 10.1007/978-3-030-26369-0_34. 